\title{
INSTRUMENTO DE AVALIAÇÃO DO ESTRESSE NA EQUIPE DE ENFERMAGEM
}

\author{
STRESS EVALUATION INSTRUMENT IN NURSING TEAM
}

\section{Joseany da Silva Santana $a^{a^{*}}$, Jorge Luiz Lima da Silva $a^{b^{* *}}$, Gabriel de Moura Mello ${ }^{c^{*}}$, Pedro Antonio Alves Bezerra Bortolazzo ${ }^{\mathrm{d}^{*}}$, Luiz Carlos dos Santos Bento ${ }^{\mathrm{e}^{*}}$, Angélica Boaventura de Souza ${ }^{*}$}

\author{
ajoseanyenf@hotmail.com, bjorgeluizlima@gmail.com, cgabrielmmello12@gmail.com, \\ dpabortolazzo@gmail.com, eluizc.be@gmail.com, fangelboasouza25@outlook.com \\ *Universidade Federal Fluminense - Rio de Janeiro (RJ), Brasil \\ **Fundação Instituto Oswaldo Cruz - Rio de Janeiro (RJ), Brasil
}

Data de recebimento do artigo: 19/12/2016

Data de aceite do artigo: 29/06/2017

\section{RESUMO}

Introdução: As equipes de enfermagem estão constantemente expostas a situaçôes que geram elevado nível de estresse, seja por conta de intercorrências médicas e incorporaçáo de novas tecnologias no atendimento aos pacientes, seja pela necessidade de se manter no mercado de trabalho, entre outras. Este estudo visa a identificar escalas/instrumentos validados e adaptados que avaliam o grau de estresse no trabalho de equipe de enfermagem no Brasil. Método: Pesquisa descritiva com revisão sistematizada da literatura em bases científicas dos oito últimos anos. Resultados: Percebeu-se que a maioria dos profissionais de enfermagem está concentrada em hospitais e mais exposta a agentes estressores. As escalas de estresse são, na atualidade, instrumentos que auxiliam na tomada de decisão e implantação de estratégias de mitigação. Conclusão: Os resultados indicam a aplicação de seis escalas e a importância da mensuração dos fatores estressores precocemente, bem como a necessidade de buscar soluçôes, refletindo na melhoria da qualidade de vida e da assistência prestada.

Palavras-chave: Equipe de enfermagem; estresse; escalas de estresse; saúde do trabalhador.

\section{ABSTRACT}

Introduction: Nursing teams are constantly exposed to situations that generate a high level of stress, due to medical intercurrences, the incorporation of new technologies in patient care, or the need to stay in the labor market, among others. This study aims to identify validated and adapted scales/instruments that assess the degree of stress in nursing work in Brazil. Method: Descriptive research with systematized review of the literature, on scientific bases of the last eight years. Results: It was noticed that the majority of nursing professionals are concentrated in hospitals, these are more exposed to stressors. Stress scales are, at present, instruments that aid in decision making, and in the implementation of mitigation strategies. Conclusion: The results indicate the application of six scales and the importance of the measurement of stressors early, as well as the need to seek solutions, reflecting on the improvement of the quality of life and care provided.

Keywords: Nursing, team; stress; stress scales; worker health. 


\section{Introdução}

O processo de crescimento tecnológico e a evoluçáo social, decorrentes da globalização, provocaram mudanças na sociedade e no estilo de vida das pessoas, transformaçóes as quais estão ligadas ao trabalho. Com a chegada do capitalismo, houve mudanças na maneira como se trabalhava, criou-se a hierarquização de cargos, submissáo dos empregados em relaçáo aos empregadores e patrôes. Não só o momento histórico e o contexto socioeconômico devem ser levados em consideraçáo para compreender o estresse ocupacional da equipe de enfermagem, também é importante distinguir o indivíduo e seu comportamento perante a dinâmica laboral.

As demandas do mercado de trabalho, hoje mais competitivo e globalizado, podem gerar, durante as atividades laborais, situaçóes reconhecidas como estressantes. Somam-se a isso fatores que envolvem o modo como o trabalho acontece e é organizado ${ }^{1}$. Além desses fatores, o ambiente de trabalho pode ser considerado um local desgastante para o profissional de enfermagem, visto que, durante seu período de trabalho, lida com dor, sofrimento e óbito de pacientes, e ainda é submetido a um sistema organizacional hierarquizado com normas rígidas, deparando com inúmeras situaçóes potencialmente estressantes.

A Organização Mundial da Saúde (OMS) aponta que os trabalhadores dos sistemas de saúde do mundo todo estáo sofrendo aumento de estresse e insegurança, na medida em que reagem a um conjunto complexo de forças ${ }^{2}$. O estresse laboral se tornou, atualmente, uma importante fonte de preocupação, e é reconhecido como risco ao bem-estar psicossocial e o bom funcionamento das instituições, resultando em alta rotatividade, aumento do absenteísmo, menor dedicaçáo ao trabalho, queda da produtividade, rendimento ruim e conflitos ${ }^{3}$. Os principais fatores geradores de estresse presentes no ambiente laboral envolvem aspectos de organização, administração do sistema de trabalho e qualidade das relações humanas. $\mathrm{O}$ fenômeno pode ser entendido como mudança imposta pela situação externa, tentativa frustrada de lidar com a situação ou circunstância, e ainda pode ser definido como situação de muita tensão ${ }^{4}$.

Nesse contexto, a enfermagem é classificada como a quarta profissão mais estressante no setor público ${ }^{5}$. Soma-se a isso o fato de a maioria dos trabalhadores de enfermagem no Brasil estar concentrada em hospitais, apresentando tendência assistencialista do setor saúde. No Brasil, é crescente o uso de escalas validadas para avaliação do estresse no trabalho, o que as tornam foco deste estudo.
O trabalho mostra-se como algo positivo, uma vez que promove crescimento, transformação, reconhecimento e independência financeira. Com isso, verificar a presença de estresse no ambiente de trabalho não deve ser tarefa fácil. Diante do exposto, este estudo levanta a questão: quais escalas avaliam o estresse no trabalho da equipe de enfermagem descritas na literatura? E tem como objetivo descrever as principais escalas/instrumentos validados, e adaptados no Brasil para avaliação do estresse no trabalho entre esses trabalhadores.

\section{Metodologia}

Pesquisa de natureza descritiva realizada por meio de revisão sistematizada da literatura, baseada em obras secundárias que abordam o tema em questão, publicadas no período de 2009 a 2016. A coleta de material para a pesquisa foi realizada no período de maio de 2016 a setembro do mesmo ano.

O levantamento foi realizado em ambiente virtual, na Biblioteca Virtual de Saúde (BVS), nas bases: Lilacs, BDENF, SciELO e em busca livre de textos completos, incluídos nos resultados com as seguintes palavras-chave: "Enfermagem"; "Estresse"; "Escalas de estresse"; "Saúde do trabalhador". Os termos foram utilizados de forma conjunta e isolados. As obras idênticas e/ou repetidas em bases diferentes foram eliminadas, e considerou-se o primeiro registro.

Foram selecionados para este estudo somente artigos que demonstrassem, na leitura, relação direta com o tema abordado. Foram incluídos periódicos da área de enfermagem publicados no Brasil, entre outros. Primeiramente, as obras foram armazenadas no computador, e, em seguida, foi realizada pré-seleção, por meio da leitura dos resumos. Nessa fase, buscou-se relação entre o conteúdo, o título e o resumo, e se estes atendiam ao objeto do estudo.

Tendo em vista o objeto da pesquisa, durante a busca, ao utilizar os descritores "Enfermagem" e "Escalas de estresse" foram encontrados 30 artigos. Desses, 6 se adequavam aos parâmetros estabelecidos. Foram descartados 24, por não caracterizarem os sujeitos. Em busca livre de textos completos, atendendo aos critérios científicos na internet, foram selecionados seis artigos em concordância com a relevância do tema abordado.

Com atenção especial para os resultados e conclusão das obras. Os trabalhos que não apresentavam qualquer relação com o estresse da equipe de enfermagem foram excluídos. Realizada triagem das obras, foram obtidos nove artigos, somados a um livro para embasamento teórico, um arquivo de outra categoria (manual). $\mathrm{Na}$ fase de seleção, as obras foram lidas na íntegra. 
Depois das etapas descritas, foram construídos nos resultados itens que abordam o estresse no trabalho da equipe de enfermagem. $\mathrm{Na}$ fase de interpretação, as obras foram lidas e analisadas.

\section{Resultados}

Para melhor visualização dos resultados, foi elaborada tabela, em que os artigos foram alocados.

Tabela 1: Publicações selecionadas para discussão, capturadas nas bases BVS e SciELO, publicadas entre 2009 e 2016.

\begin{tabular}{|c|c|c|c|c|}
\hline Autores & Objetivo da pesquisa & $\begin{array}{c}\text { Método/Tamanho da } \\
\text { amostra/Tipo de estudo }\end{array}$ & Instrumento & Conclusão do artigo \\
\hline $\begin{array}{l}\text { Schmidt, Dantas, } \\
\text { Marziale e Laus }{ }^{6}\end{array}$ & $\begin{array}{l}\text { Avaliar presença de } \\
\text { estresse ocupacional entre } \\
\text { profissionais de enfermagem } \\
\text { que atuam nos blocos } \\
\text { cirúrgicos. }\end{array}$ & $\begin{array}{l}\text { Descritivo e correlacional, } \\
\text { de corte transversal. } \\
\text { Com } 211 \text { trabalhadores } \\
\text { de enfermagem do bloco } \\
\text { cirúrgico. }\end{array}$ & Job Stress Scale & $\begin{array}{l}56,1 \% \text { exposição intermediária ao estresse } \\
\text { Ocupacional; } 26,3 \% \text { alta exposição ao } \\
\text { estresse e } 17,6 \% \text { sem exposiçáo ao estresse. }\end{array}$ \\
\hline Bianchi $^{7}$ & $\begin{array}{l}\text { Discutir sobre o estresse do } \\
\text { enfermeiro hospitalar. }\end{array}$ & Revisão de literatura. & $\begin{array}{l}\text { Escala Bianchi de } \\
\text { Stress (EBS) }\end{array}$ & $\begin{array}{l}\text { A EBS é um instrumento de análise da } \\
\text { variação do nível de estresse dos enfermeiros } \\
\text { em cada tipo de unidade de atuação em } \\
\text { instituição hospitalar. }\end{array}$ \\
\hline $\begin{array}{l}\text { Farias, Teixeira, } \\
\text { Moreira, Oliveira } \\
\text { e } \text { Pereira }^{5}\end{array}$ & $\begin{array}{l}\text { Identificar os fatores } \\
\text { desencadeantes de estresse } \\
\text { na equipe de enfermagem } \\
\text { que trabalha na unidade de } \\
\text { Pronto Atendimento. }\end{array}$ & $\begin{array}{l}\text { Quantitativo/Qualitativo. } \\
\text { Com } 21 \text { trabalhadores de } \\
\text { enfermagem do Pronto } \\
\text { Atendimento. }\end{array}$ & $\begin{array}{l}\text { Occupational Stress } \\
\text { Indicator (OSI) }\end{array}$ & $\begin{array}{l}\text { Os sintomas físicos caracterizadores de } \\
\text { estresse foram: cefaleia, sensaçáo de fadiga, } \\
\text { dores nas pernas e taquicardia. }\end{array}$ \\
\hline $\begin{array}{l}\text { Griep, Rotenberg, } \\
\text { Landsbergis e } \\
\text { Vasconcellos-Silva }\end{array}$ & $\begin{array}{l}\text { Identificar combinaçóes de } \\
\text { dois modelos de estresse } \\
\text { psicossocial do trabalho em } \\
\text { equipes de enfermagem e } \\
\text { sua associaçáo com a saúde } \\
\text { autorreferida. }\end{array}$ & $\begin{array}{l}\text { Transversal. } \\
\text { Com trabalhadores de três } \\
\text { hospitais públicos. }\end{array}$ & $\begin{array}{l}\text { Modelo } \\
\text { demanda-controle } \\
\text { (DC) e desequilíbrio } \\
\text { esforço-recompensa } \\
\text { (DER) }\end{array}$ & $\begin{array}{l}\text { Tiveram maior desempenho do modelo DER } \\
\text { com esse grupo específico de trabalhadores, } \\
\text { pois esse modelo é fundamentado na } \\
\text { reciprocidade entre o esforço gerado nas } \\
\text { tarefas e a compensaçáo em termos de salário, } \\
\text { segurança no trabalho, perspectivas e estima. }\end{array}$ \\
\hline $\begin{array}{l}\text { Lima, Simonett, } \\
\text { Bianchi e } \\
\text { Kobayashi }^{1}\end{array}$ & $\begin{array}{l}\text { Caracterizar o estresse dos } \\
\text { enfermeiros que atuam em } \\
\text { Cardiologia. }\end{array}$ & $\begin{array}{l}\text { Quantitativa, descritiva } \\
\text { e comparativa. } \\
\text { Participaram da pesquisa } \\
77 \text { enfermeiros. }\end{array}$ & $\begin{array}{l}\text { Foram utilizadas as } \\
\text { escalas EBS, ETT e } \\
\text { Estresse Percebido } \\
\text { (PPS) }\end{array}$ & $\begin{array}{l}\text { Os enfermeiros apresentam nível médio de } \\
\text { estresse e as maiores causas são administração } \\
\text { de pessoal, condiçóes de trabalho e } \\
\text { coordenaçáo das atividades na unidade. }\end{array}$ \\
\hline
\end{tabular}

\section{Discussão}

$\mathrm{Na}$ literatura nacional, ainda é tímida a produção envolvendo estresse entre equipe de enfermagem. Foram encontrados seis artigos sobre a temática, sendo dois de 2009, três de 2011 e um de 2012. As pesquisas foram realizadas em cenários variados de pesquisa: pronto atendimento, setor de cardiologia, bloco cirúrgico e hospitais.

Tabela 2: Relação das escalas aplicadas encontradas em artigos científicos nas bases virtuais entre 2009 e 2016 .

\begin{tabular}{|ll|}
\multicolumn{1}{|c}{$\begin{array}{c}\text { Escalas de estresse das } \\
\text { equipes de enfermagem }\end{array}$} & \multicolumn{1}{c}{$\begin{array}{c}\text { Quantidade de } \\
\text { artigos encontrados }\end{array}$} \\
\hline Demanda-controle/Job Stress Scale (JSS) & 3 artigos \\
\hline Escala de Estresse no Trabalho (EET) & 2 artigos \\
\hline Escala Bianchi de Stress & 2 artigos \\
\hline
\end{tabular}

continua..
Tabela 2: Continuação.

\begin{tabular}{|c|c|}
\hline $\begin{array}{l}\text { Escalas de estresse das } \\
\text { equipes de enfermagem }\end{array}$ & $\begin{array}{c}\text { Quantidade de } \\
\text { artigos encontrados }\end{array}$ \\
\hline Desequilíbrio esforço-recompensa (DER) & 1 artigo \\
\hline Indicador de estresse ocupacional & 1 artigo \\
\hline Escala de Estresse Percebido (PPS) & 1 artigo \\
\hline
\end{tabular}

Fonte: Elaborado pelos autores (2017).

\section{Descrição das escalas}

Robert Karasek foi um dos primeiros pesquisadores a se interessar pelos fatores que desencadeiam o estresse no trabalho e suas repercussóes na saúde. Por volta de 1970, propôs o modelo que considerava a demanda e controle no trabalho (ritmo/intensidade e autonomia/ habilidade necessária) relacionados ao risco de adoecimento. Esse modelo teórico bidimensional, quando em 
desequilíbrio, favorece o desgaste no trabalho (job strain) e o consequente adoecimento, sendo necessárias açóes mitigadoras no ambiente de trabalho ${ }^{10}$. Posteriormente, o modelo passou a incluir uma terceira percepçáo, a do apoio social no trabalho, com contribuiçấo dos estudiosos Johnson e Hall. A dimensão apoio social integra a confiança no grupo, ajuda na realização das tarefas por parte dos colegas e superiores, os quais poderiam atuar como moderadores dos efeitos do desgaste no trabalho sobre a saúde ${ }^{11}$. Em 1988, na Suécia, Töres Theorell desenvolveu versóes traduzidas e adaptadas para o português brasileiro do questionário de Karasek (49 perguntas), o Job Content Questionnaire e a Escala Reduzida, denominada Escala Sueca de demanda-controle-apoio social (DCS). Também contendo versão para o português, a Job Stress Scale (JSS) contém 17 questóes: cinco para avaliar demanda, seis para avaliar controle e seis para apoio social.

Desenvolvida em 1988, o Occupational Stress Indicator (OSI) feito por Cooper, Sloan e Williams, aborda as medidas de estressores, mediadores e consequências físicas e psicológicas no processo do estresse ocupacional. Ao todo, são 167 itens agrupados em 25 fatores. Os itens do OSI foram traduzidos para a língua portuguesa por Swan, Moraes e Cooper em 1993. Assim como existe a validação desse instrumento no Brasil, faltam dados sobre sua adequação, e a validação tem recebido críticas ${ }^{12}$.

Em 1983, Cohen e outros pesquisadores propuseram uma escala que mensurasse o estresse percebido, ou seja, que medisse o grau no qual os indivíduos percebem as situações como estressantes. Essa escala, denominada Perceived Stress Scale (Escala de Estresse Percebido, PSS), foi apresentada inicialmente com 14 itens (PSS 14), sendo também validada com dez (PSS 10) e quatro questôes (PSS 4) ${ }^{13,14}$. A versão com quatro questóes normalmente é utilizada em pesquisas por telefone, com objetivo de verificar o quanto imprevisível, incontrolável e sobrecarregada os respondentes avaliam suas vidas. Esses três fatores têm sido considerados instrumentos principais na sintomatologia do estresse. A PSS é uma escala geral, que pode ser usada em diversos grupos etários, de adolescentes a idosos, pois não contém questóes específicas de contexto. A ausência de questóes específicas de contexto é fator importante na escala e, provavelmente, a razáo pela qual a escala tenha sido validada em diversas culturas ${ }^{14}$.

Os itens da Escala de Estresse no Trabalho (EET) foram elaborados a partir dos estressores organizacionais de natureza psicossocial, e das reaçóes psicológicas ao estresse ocupacional, além de analisarem instrumentos existentes. Cada item da EET aborda tanto estressor quanto reação ao fenômeno, sugerido em 1987 por Murphy e Hurrel ${ }^{15}$. Estressor pode ser entendido como qualquer evento, interno ou externo, que leve o indivíduo a avaliação cognitiva desse evento. Lidar com eventos extremos, como a vida e a morte, podem representar, para a equipe de enfermagem, um estressor que, na avaliação do modelo intervencionista, pode ser interpretado como desafio.

O modelo desequilíbrio esforço-recompensa (Effort-Reward Imbalance Model, ERI) foi desenvolvido na década de 1990, onde o excesso de esforço no trabalho e o baixo reconhecimento eram geradores de situaçôes estressantes. O esforço está relacionado às demandas de trabalho e obrigaçóes percebidas pelo trabalhador: a recompensa, por salário adequado; autoestima profissional; respeito e apoio por parte dos colegas e superiores; perspectivas de promoção, estabilidade no emprego, e status social. Houve então, a necessidade de inclusão de uma terceira dimensão ao modelo original: "o excesso de comprometimento com o trabalho", expresso no empenho exagerado no trabalho por parte do trabalhador, combinado com um forte desejo de ser aprovado e estimado (status social). Esse instrumento encontra-se validado no Brasil por Guimarães, Zaroni e Martins, que avaliam as dimensões "esforço", "recompensa" e "excesso de compromisso". A avaliaçáo é um questionário, composto por 57 questóes do tipo Likert (escala psicométrica), divididas em três partes: a primeira analisa alguns dados sociodemográficos e a situação objetiva de trabalho; por exemplo, tipo de trabalho, turnos, tipo de organização que trabalha, entre outros; a segunda avalia a percepção do sujeito quanto à situação no trabalho; e a terceira avalia aspectos subjetivos do sujeito em relação à características intrínsecas de "supercomprometimento" com o trabalho, denominada "superexigência"16,17.

A Escala Bianchi de Stress foi construída e validada para avaliar o nível de estresse do enfermeiro hospitalar no desempenho básico de sua função. É autoaplicável, composta por 51 itens, divididos em seis domínios: relacionamento com outras unidades e supervisores (A); funcionamento adequado da unidade (B); administração de pessoal (C); assistência de enfermagem prestada ao paciente (D); coordenação das atividades (E) condições de trabalho $(\mathrm{F})^{7}$. As questóes recebem pontuação com variação de 1 a 7 , sendo: baixo (até 3), médio $(3,1$ a 4,0$)$, alerta $(4,1$ a 5,9) e alto (acima de 6). É um instrumento que auxilia na tomada de decisão para a implantação de estratégias de enfrentamento do estresse do enfermeiro hospitalar, e também apresenta uma variação do nível de estresse em cada tipo de unidade de atuação.

Esses são os instrumentos validados para avaliar o estresse, e os estudos apontam para alto grau de estresse, nas relações da equipe de enfermagem no ambiente de trabalho. 


\section{Conclusão}

As escalas de estresse são hoje instrumentos que auxiliam na tomada de decisão para implantação de estratégias de enfrentamento do estresse, nas relaçóes da equipe de enfermagem. Sabe-se ainda que a maioria dos hospitais passa por carência de recursos, com número reduzido de profissionais, carga horária elevada, produçáo de tarefas em tempo reduzido, conflito de funçóes e desvalorização do trabalho.

$\mathrm{Na}$ maioria dos hospitais, o trabalho da equipe de enfermagem tem sido apontado como altamente estressante, podendo ser justificado pelo alto nível de responsabilidade e baixa autonomia, que refletem situaçôes com vários pontos de tensão. É necessário discutir as condições de trabalho desse profissional, independentemente de sua área de atuação. A própria equipe precisa reconhecer que a profissão é estressante, e desmistificar que o estresse é fruto somente da pessoa, esquecendo-se da organização do trabalho.

As relaçôes interpessoais devem ser foco de açóes para redução do estresse dos enfermeiros, e é preciso investir na valorizaçáo para atingir uma assistência de qualidade com excelência.

Este estudo mostra que, nos últimos oito anos, seis escalas vêm sendo aplicadas para mensuraçáo do estresse, as quais foram identificadas nas bases virtuais, ganham destaque: Job Stress Scale; Escala de Estresse no Trabalho; e, Escala Bianchi de Stress.

\section{Referências}

1. Lima FG, Simonetti SH, Bianchi ERF, Kobayashi RM. Caracterização do estresse de enfermeiros que atuam em hospital especializado em cardiologia. Enfermería glob. 2012;11(28):105-119.

2. Organização Mundial Da Saúde. Trabalhando juntos pela saúde: Relatório Mundial de Saúde 2006. Brasília, DF: Ministério da Saúde; 2007.

3. Rossi AM. Estressores ocupacionais e diferenças de gênero. In: Rossi AM, Perrewé PL, Sauter SL. Stress e qualidade de vida no trabalho: perspectivas atuais da saúde ocupacional. São Paulo: Atlas; 2005.
4. Corrêa RZA, Souza MS, Baptista MN. Vulnerabilidade ao estresse no trabalho e a qualidade de vida de enfermeiros. Psicol argum. 2013;31(75):599-606.

5. Farias SMC, Teixeira OLC, Moreira W, Oliveira MAF, Pereira MO. Caracterização dos sintomas físicos de estresse na equipe de enfermagem de pronto atendimento. Rev Esc Enferm USP. 2011;45(3):722-29.

6. Schmidt DRC, Dantas RAS, Marziale MHP, Laus AM. Estresse ocupacional entre profissionais de enfermagem do bloco cirúrgico. Text. Cont. Enferm. 2009;18(2): 330-37.

7. Bianchi ERF. Escala de Bianchi de Stress. Rev. esc. enferm. USP. 2009;43(Esp):1055-62.

8. Costa DT, Martins MSF. Estresse em profissionais de enfermagem: impacto do conflito no grupo e do poder do médico. Rev. Esc. Enferm. USP. 2011;45(5): 1191-98.

9. Griep RH, Rotenberg L, Landsbergis P, Vasconcellos-Silva PR. Uso combinado de modelos de estresse no trabalho e a saúde auto-referida na enfermagem. Rev. Saud. Public. 2011;45(1):145-52.

10. Alves MGM, Chor D, Faerstein E, Lopes CS, Werneck GL. Versão resumida da "job stress scale": adaptação para o português. Rev. Saúd. Public. 2004;34(2):164-71.

11. Johnson JV, Hall EM. Job strain, workplace social support and cardiovascular disease: a cross-sectional study of a random sample of the Swedish working population. Am J Public Health. 1988;78(10):1336-42.

12. Oliveira DFL. O uso de escalas para mensurar o grau de estresse em trabalhadores de enfermagem. Rio de Janeiro. Monografia [Especilização em Enfermagem do Trabalho] Universidade Federal Fluminense; 2011.

13. Cohen S, Kamack T, Mermelstein R. A global measure of perceived stress. J Health Soc Behav. 1983;24(4):385-96.

14. Luft CB, Sanches SO, Mazo GZ, Andrade A. Versão brasileira da Escala de Estresse Percebido: Tradução e validação para idosos. Rev. Saúde Públ. 2007;41(4):606-15.

15. Paschoal T, Tamayo A. Validação da escala de estresse no trabalho. Estud. Psicol. 2004;9(1):45-52.

16. Chor D, Werneck GL.; Faerstein E, Alves MGM, Rotenberg L. The Brazilian version of the effort-reward imbalance questionnaire to assess job stress. Cad. Saúde Pública. 2008;24(1):219-224.

17. Vasconcelos EF, Guimarães LAM. Esforço e recompensa no trabalho de uma amostra de profissionais de enfermagem. Psicólogo in Formação. 2009;13(13):11-36.

\section{Como citar este artigo:}

Santana JS, Lima da Silva JL, Mello GM, Bortolazzo PAAB, Bento LCS, Souza AB. Instrumento de avaliação do estresse na equipe de enfermagem. Rev. Aten. Saúde. 2017;15(52):61-65. 